\title{
THREAT TO ASTRONOMY BY SPACE ART
}

\author{
PAUL MURDIN \\ Royal Greenwich Observatory \\ Herstmonceux Castle, Hailsham, East Sussex BN27 1RP, U.K.
}

\begin{abstract}
The Light Ring, proposed as a celebration of the centenary of the Eiffel Tower, and now withdrawn by its sponsors, demonstrated the feasibility to launch relatively inexpensive satellites which subtend an appreciable solid angle as seen from earth. Other satellites in the competition organized by the Eiffel Tower proposed configurations under the name of Space Art which could unacceptably increase the night sky background. Satellites of this nature, launched for noble but essentially frivolous motives, pose a threat to optical astronomy.
\end{abstract}

\section{INTRODUCTION}

Several contributors in yesterday's session expressed support for astronomy in combating pollution, although they themselves were engineers and amateurs. They too had a love of this science.

Just as some people are astronomers, so also some astronomers are people. I have a family whom I wish to be safe as they walk in London at night. I drive a car and I wish not to injure someone with it because I did not see them. I used the phone yesterday to call home, probably through a satellite channel. I also respond to expressions of art and support artists in their endeavors to show us fresh ways of seeing our surroundings. However, my support for art does not mean that I must refrain from criticizing questionable projects, just as I urge that care be taken to control lighting and space junk.

\section{BRIGHT SATELLITES AND THE NIGHT SKY BACKGROUND}

The present population of artificial earth satellites (Kessler, this volume) at an altitude of $500 \mathrm{~km}$ is at nearly all sizes from 1 micron to 10 meters more dense than the natural background of meteors. The density of large satellites (size greater than $1 \mathrm{~cm}$ ) is especially greater than the natural background. Such objects are the ones which are most readily detected by optical astronomers.

Depending, of course, on albedo, reflection properties, phase angle and distance, satellites of $1 \mathrm{~cm}$ diameter and larger are brighter than magnitude 18 . The standard satellite magnitude for a diffusely reflecting spherical satellite at $1000 \mathrm{~km}$ range, at phase 0.5 is approximately 


$$
\mathrm{m}=+5-5 \log (\mathrm{D} / 3.6)
$$

where the diameter is $\mathrm{D}$ meters; the expression is calibrated relative to Explorer 19, which was $3.6 \mathrm{~m}$ diameter and magnitude +5 .

At what size does a satellite become a significant contributor to the night sky brightness? Optical observations divide the month into "bright time," "grey time," and "dark time," depending on the phase of the Moon. Because dark time has the minimum light from the night sky, it is highly sought after for the observation of faint objects for which the noise of the background contribution is minimized. The study of faint objects is significant, for example, in cosmology, since "fainter" is in general equivalent to "more distant" and therefore in the time in the past when the light being studied was emitted. The magnitude of the Moon and brightness of the sky which it causes is listed in Table 1.

\section{TABLE I. NIGHT SKY BRIGHTNESS}

\begin{tabular}{llccc} 
& & Mag. & $\begin{array}{l}\text { Night-sky } \\
\text { brightness } \\
\text { (mag/sq") }\end{array}$ & \\
& & & & \\
Bright time & Full Moon & -13 & 17.7 & low \\
Bright time & 5d off Full Moon & -11 & 18.9 & low \\
Grey time & 9d off Full Moon & -9 & 21.4 & med \\
Dark time & New Moon & $(-8)$ & 22.0 & high \\
\hline
\end{tabular}

From Eqn (1) it is clear that diffusely reflecting structures that have a diameter in excess, say, of $1000 \mathrm{~m}$ will unacceptably increase the brightness of the night sky. Such structures belong to the rather distant future.

Smaller structures which reflect specularly and which focus light on the earth may cause local brightening of the night sky. The concentration factor is $\Omega / 4 \pi$ where $\Omega$ is the solid angle subtended by the illuminated region at the satellite. Illumination of a region $500 \mathrm{~km}$ in diameter from a focussed satellite at $1000 \mathrm{~km}$ range is 50 times brighter than illumination by an isotropic diffuser. The brightness of the night sky background within the illuminated region is increased in the same proportion.

\section{CONFUSION AND DAMAGE}

Maley (this conference) gave examples of confusion for astronomy resulting from flashing satellites. Satellite trails confuse observations by obscuring regions of the detector field. In the case of bright satellite trails on small area detectors the observation must be rejected. The effect of the trail is diluted by the ratio of the time of passage of the satellite across a detector element to the exposure time. As detectors become more efficient and exposure times become shorter the effects of trails become more severe. 
The probability of catching a confusing trail increases with the field of view of the detector. Wide angle Schmidt telescopes are most vulnerable. During the taking of the UK Schmidt Survey in the mid 1970s, plates were initially rejected if they contained any satellite trails, but this proved difficult to continue, and some plates with satellite trails had to be accepted as "survey quality." Plates with trails that were confusing were rejected: criteria for rejection included brightness, passage across notable regions, interrupted trails, or number of trails. Brucato (this conference) told us that purchasers of the new Palomar Sky Survey presently being exposed will have to accept fields with trails. At the UK Schmidt at the present time every exposure made within an hour or two of sunrise or sunset (45 min exposures, 60 field of view) contains a satellite trail, with an average of 5 .

Telescopes with smaller fields of view may use compensatingly more sensitive detectors. During a night in which I acted as a night assistant on the 4.2 meter William Herschel Telescope, I counted five bright objects trailing across the 2 arc-minute field of the slit viewing TV, most of which were probably satellites. The TV system can view to a magnitude of 22 or so, with integration times which vary with telescope use but which are typically several seconds to a minute. The magnitudes of the satellites seen are unknown.

Satellites that cause continuous trails are recognizable on area detectors. It is more difficult to distinguish sporadic flashers. Regular flashers which produce interrupted trails across Schmidt telescope plates can be identified. The appearance of their individual flashes (if they are brief) can be startlingly like elliptical galaxies. Specular reflections of very brief duration, or geostationary satellites, or other satellites with low angular speed also produce astronomicallooking trails. The number of such spurious images on Schmidt plates has not been studied.

Astronomers can anticipate confusion from space debris in studies such as surface photometry, search for optical bursts, surveys for variability, and surveys for color.

As documented by Taff (this conference) the space debris problem for optical astronomers extends beyond the 7000 satellites listed in the catalog. To magnitudes detectable by optical astronomers there are up to $10^{5}$ artificial earth satellites.

If faint satellites are responsible only for spoiling observations by confusion, bright satellites may actually damage equipment. Photographic and CCD cameras are not vulnerable to damage by bright lights, but intensifier-based detectors are. The Image Photon-Counting System (IPCS) is in use on La Palma, at the Anglo Australian Telescope and at the Palomar Observatory, using a high gain $\left(x 10^{7}\right)$ intensifier as a front end, amplifying individual photons for subsequent detection. Intense light deposits so much energy in the phosphors of the intensifier or its photocathodes that they may be physically destroyed. The intensifiers used are selected for astronomical use (uniformity, low dark count, red sensitivity) and there exist only a dozen suitable tubes worldwide.

The magnitude of a satellite which will damage an intensifier depends on the size of the telescope and instrument on which it is being used (Table 2). The predominant use of the IPCS is as a spectroscopic detector, with an entrance aperture of approximately $200 \mathrm{sq}$ arc sec aperture.

Its use as an area detector exposed directly to the sky (for example, the high time-resolution detection of the Vela pulsar, Peterson et al. 1978) must now be 
regarded as risky, in view of the number of artificial satellites of the damaging brightness.

Table II. DAMAGE THRESHOLD FOR IPCS

Telegcope Satellite Magnitude at which damage occurs

A. IPCS used as a spectroscopic detector (1A resolution)
$2.5 \mathrm{~m}$
$-1$
$10 \mathrm{~m}$
$+2$

B. IPCS used as an area detector (1000 A bandpass)

$2.5 \mathrm{~m}+9$

$10 \mathrm{~m}+12$

\section{LARGE BRIGHT SATELLITES AND DISPLAY SATELLITES}

The largest satellites whose launch is agreed range up to the $100 \mathrm{~m}$ class (taking account of the size of the solar panels deployed to increase their surface area). Such satellites will increase the population of bright satellites, and will rival the luminosity of the bright planets. Specular reflections from such satellites will be problematical as will their potential for interference with observations.

Other large satellites can be envisaged (space colonies for example). Closer to us in practicality is a new class of "display satellites." These are subdivided into three kinds:

a) Art Satellites. Several proposals have been made for the launch of structures as works of art. These have noble purposes: they cause mankind to review its attitude to the sky which they adorn, like a piece of jewelry which enhances a beautiful face. The enthusiasm for art satellites (ARSATS) has led to the proposal for the next kind of display satellites.

b) Commemorative Satellites. These are best described by one of their enthusiasts (P. Comte, 1986): Each time an event of worldwide significance takes place (Olympic games, international expositions, anniversaries important to our planet), it would be possible to announce it, to celebrate it by means of the presence of the ARSAT satellites parading on the vault of heaven: a mobile star of extreme brightness.

c) Advertising Satellites. Just as creation of artistic images has been adopted by the advertising industry for magazines, TV, posters, etc., we may expect proposals for the advertising use of satellites. The TV advertising industry measures advertising impact in terms of exposure-minutes. An advertising satellite visible to the population of the world on several occasions per year seems to be cost-effective in these terms.

All these aspects of display satellites were present in the proposal by the Eiffel Tower Company in 1987 to celebrate its centenary by launching a Light Ring (Murdin, 1987). 
The Light Ring was proposed to be an inflatable structure which at a typical range would appear as an extended object of diameter 0.5 degree. This would have been the first satellite with appreciable angular extent, all previous objects being unresolved when conventionally viewed from the ground. The probability that such a satellite of apparent diameter $\phi_{g}$ would intrude on the field of view of a detector $\phi_{\mathrm{d}}$ in a single pass overhead is approximately

$$
p=180\left(\phi_{s}+\phi_{d}\right) / 20000 \mathrm{sq} \text { deg }
$$

where $20000 \mathrm{sq} \mathrm{deg}$ is the area of a hemisphere. The vulnerable periods for a telescope are within an hour or two of sunrise and sunset and while the telescope is actually exposing. Taking into account the known characteristics of a typical efficient telescope such as the Isaac Newton Telescope (Benn \& Martin, 1987) the probability that a satellite will intrude into the field of view can be calculated (Table III).

\section{TABLE III PROBABILITY OF VIEWING A SATELLITE BY CHANCE}

$\begin{array}{ccc}\text { Detector field } & \begin{array}{c}\text { Probability of } \\ \text { intrusion in a } \\ \text { single pass }\end{array} & \begin{array}{c}\text { Probability of } \\ \text { intrusion in }\end{array} \\ & \begin{array}{c}1 \text { year } \\ \end{array} & \end{array}$

A. Point-like satellite $\phi_{\mathbf{g}}=0$ deg

$\begin{array}{ccc}1 . & 1.5 \times 10^{-4} & 4 \times 10^{-3} \\ 1 . & 1 \times 10^{-2} & 0.2 \\ 6^{.} & 5 \times 10^{-2} & 1\end{array}$

B. Extended satellite $\phi_{\mathrm{s}}=0.5 \mathrm{deg}$
1.
$5 \times 10^{-3}$
0.1

For point-like satellites, the probability of intrusion into typical fields for spectroscopy ( $\phi_{\mathrm{d}}=$ the square root of $200 \mathrm{arcsec}$ ), over the decade that modern detectors have existed, has been small; we have evidently been lucky in that there has been no known case of a detector being destroyed by satellite intrusion. If extended satellites are launched, it will be necessary to take measures to avoid accidental exposure to them.

It will likely be difficult to protect telescopes by schedule (time sharing), without considerable effort. Extended satellites will be subject to radiation pressure from the Sun and Earth, particle pressure from the Sun, and atmospheric drag, all of which will depend in a complex way on geometry. A low mass/area spherical satellite like Pageos is subject to 15 minute variations on orbital predictions made 3 weeks in advance, and a large asymmetric satellite is likely to be more unpredictable. Thus, guard systems will be necessary, at first no doubt four students viewing $\mathrm{N}, \mathrm{S}, \mathrm{E}$ and $\mathrm{W}$, ready to shout a warning, and later some more sophisticated automatic system. 


\section{CONTROL OF ART SATELLITES}

Regulation of space satellites is by the individual space agencies (NASA, ESA, etc.). While there are a few internationally agreed restrictions on the use of space (tests of nuclear weapons being one forbidden category), the regulatory agencies require space verification of payloads before launch. Consultative procedures to determine the environmental impact of satellites should be part of the evaluation process. British government policy is that the use of space satellites for advertising should be controlled by an international code of conduct. If such a code is drawn up, damage limitation could be achieved by compromises such as the following:

a) minimize number and duration of displays (days, not years);

b) no focused satellites;

c) minimum (zero) angular extent;

d) orbits chosen for predictability and minimum impact on astronomy (e.g. twilight visibility).

In fact, these conditions would so limit the display that it may be unattractive to launch display satellites. If the display is so prominent that it is worth being called space art, it is probably too prominent for astronomers to accept. The best brake on this activity would be from the proposers themselves, and I make this statement to the artists:

Please take into account what the astronomers say and, if you can, find some other way to express your legitimate aspirations to comment, as we do, upon our place in the Universe.

\section{REFERENCES}

Benn, C. R. and Martin, R. M., Q.J.R.A.S., 28, 481.

Comte, p. (1986), Leonardo, 19, 17.

Murdin, P. (1987), Nature, 326, 125.

Peterson, B. A. et al. (1978), Nature, 276, 475. 